\title{
Fault Diagnosis of Rolling Bearings Based on Improved Empirical Mode Decomposition and Fuzzy C-Means Algorithm
}

\author{
Hailun Wang ${ }^{1,2 *}$, Fei $\mathrm{Wu}^{2}$, Lu Zhang ${ }^{2}$ \\ ${ }^{1}$ Logistics Engineering College, Shanghai Maritime University, Shanghai 200000, China \\ ${ }^{2}$ College of Electrical and Information Engineering, Quzhou University, Quzhou 324000, China
}

Corresponding Author Email: wanghl@qzc.edu.cn

https://doi.org/10.18280/ts.380217

Received: 15 October 2020

Accepted: 19 January 2021

\section{Keywords:}

rolling bearings, variational modal decomposition (VMD), fuzzy C-means (FCM) algorithm, fault identification

\begin{abstract}
The fault signals of rolling bearings have a very low signal-to-noise ratio (SNR), making it difficult to fully extract and reconstruct the fault signals. To solve the problem, this paper proposes a way to recognize rolling bearing faults based on improved variational modal decomposition (VMD) and fuzzy c-means (FCM) algorithm. Firstly, the measured vibration signals of rolling bearings were subject to VMD on different scales. Next, the FCM clustering was performed to classy and recognize the eigenvectors of sample signals. Then, the authors calculated the normalized energy ratio of the autocorrelation function for each model obtained by decomposition, and applied it to optimize and reconstruct modal signals. Finally, the eigenvectors of sample signals were classified and recognized through FCM clustering. Several experiments were carried out to compare the improved VMD with empirical mode decomposition (EMD) and local mean decomposition (LMD) in the fault recognition and classification of rolling bearings in different backgrounds of strong composite noises. The comparison shows that the improved VMD has a strong denoising ability; the stronger the additive noise, the more superior the improved VMD is to LMD and EMD, and the better its feature clustering effect. The experimental results fully manifest the effectiveness of the proposed method.
\end{abstract}

\section{INTRODUCTION}

During the operation of rotating machinery, the fault vibration signals are non-stationary and nonlinear, under the effects of strong background noise, structural deformation, acquisition instruments, and signal attenuation. Therefore, it is very difficult to extract the fault features of rolling bearings. To classify the faults of rolling bearing, the key is to effectively extract the fault features under strong noises [1].

The fault vibration signals measured from rolling bearings are featured by non-stationarity and nonlinearity [2, 3]. In recent years, empirical mode decomposition (EMD) and local mean decomposition (LMD) have been widely applied to extract the features for fault diagnosis [3]. However, the two recursive modal decomposition approaches face the problem of modal aliasing. They are unable to separate the components with similar frequencies. Besides, both EMD and LMD have the endpoint effect. Hence, the two approaches are susceptible to sampling frequency, and erroneous in decomposition $[4,5]$.

Yang et al. [6] utilized EMD to decompose the signal, and extracted the sensitive features of rolling bearings with the local tangent space alignment algorithm. Zolfaghari et al. [7] transformed the decomposed signals by the coef wavelet packet, searches for the maximum amplitude through fast Fourier transform, and makes the search result as a feature of bearing vibration signals. Wang et al. [8] processed the early fault signals with LMD, extracted fault frequencies by envelope spectrum analysis, and experimentally proved that their approach can effectively mine fault features. Despite their adaptability, these approaches each has its defects. For example, the EMD has serious modal aliasing, and the decomposition result is distorted by envelope error $[9,10]$.

LMD faces weaker modal aliasing than EMD, but still has the endpoint effect [11-13]. In 2014, DragomiRetskiy et al. presented a novel adaptive signal analysis method called variational modal decomposition (VMD). Through iterative search for the optimal solution to the variational model, this algorithm determines the center frequency and bandwidth of each modal, divides the signals in the frequency domain, and effectively separates different components. The VMD boasts high accuracy, fast convergence, and effective denoising effect. However, the modal components might be noise even after VMD. Therefore, modal signals with high signal-to-noise ratio (SNR) should be selected for signal reconstruction [14].

Ma et al. [15] studied the application of VMD in the fault classification of bearings, and extracted the fault features of rolling bearing by combining the signals reconstructed from the VMD results with the Teager energy operator. Mohanty [16] optimized the VMD parameters, then processed the early fault signals of rolling bearings, and succeeded in extracting weak feature information. The fuzzy c-means (FCM) algorithm, a common technique in fuzzy cluster analysis, classifies data by clustering similar data points. This algorithm has been widely adopted to detect machine faults $[17,18]$.

Through the above analysis, this paper proposes a rolling bearing fault recognition method that improves VMD with FCM. Comparative experiments were carried out on the denoising effect of our method in the context of strong composite noises. The results show that our improved VMD can effectively remove noises. Then, the method was applied 
to analyze the data of rolling bearing fault tests, and compared with EMD and LMD in clustering effect. It was learned that our method is superior and effective in recognizing rolling bearing faults.

\section{IMPROVED VMD ALGORITHM}

\subsection{VMD}

The VMD needs to preset the scale $K$, i.e., the number of bidimensional intrinsic modal functions (BIMFs) obtained through decomposition. Through an iterative process, the VMD obtains the optimal solution to the variational model, and determines the frequency centers and modal functions of the $K$ BIMFs. To evaluate the bandwidth of each modal, a variational constraint can be constructed as:

$$
\begin{aligned}
& \min _{u_{k} \cdot \omega_{k}}\left\{\sum_{k=1}^{K}\left\|\partial_{t}\left[\left(\delta(t)+\frac{j}{\pi t}\right) * u_{k}(t)\right] e^{-j \omega_{k} t}\right\|_{2}^{2}\right\}, \\
& \sum_{k=1}^{K} u_{k}(t)=f(t)
\end{aligned}
$$

where, $u_{k}$ is a BIMF; $\omega_{k}$ is the center frequency of the BIMF; $\partial_{t}$ is the search for partial derivative of time $t ; \delta(t)$ is the unit pulse function; $f(t)$ is the original signal.

To find the optimal solution, secondary penalty factor $\alpha$ and Lagrangian multiplier $\lambda$ need to be introduced to get the augmented Lagrangian function $L$ :

$$
\begin{aligned}
& L\left(u_{k}, \omega_{k}, \lambda\right)=\alpha \sum_{k=1}^{K}\left\|\partial_{t}\left[\left(\delta(t)+\frac{j}{\pi t}\right) * u_{k}(t)\right] e^{-j \omega_{k} t}\right\|_{2}^{2}+ \\
& \left\|f(t)-\sum_{k=1}^{K} u_{k}(t)\right\|_{2}^{2}+\left\langle\lambda(t), f(t)-\sum_{k=1}^{K} u_{k}(t)\right\rangle
\end{aligned}
$$

Then, the alternating direction method of multipliers (ADMM) is applied to iteratively find the minimum point of L. The specific process of the VMD can be summarized as follows:

Step 1. Initialize $\hat{u}_{k}^{1}, \omega_{k}^{1}$, and $\hat{\lambda}$, and $n \leftarrow 0$;

Step 2. $n \leftarrow n+1$, execute the loop;

Step 3. $k=1: K$, update $\hat{u}_{k}(\omega)$ :

$$
\hat{u}_{k}^{n+1}(\omega) \leftarrow \frac{\hat{f}(\omega)-\sum_{i<k} \hat{u}_{i}^{n+1}(\omega)-\sum_{i>k} \hat{u}_{i}^{n}(\omega)+\frac{\hat{\lambda}^{n}(\omega)}{2}}{1+2 \alpha\left(\omega-\omega_{k}^{n}\right)^{2}}
$$

Step 4. Update $\omega_{k}$ :

$$
\omega_{k}^{n+1} \leftarrow \frac{\int_{0}^{\infty} \omega\left|\hat{u}_{k}^{n+1}(\omega)\right|^{2} d \omega}{\int_{0}^{\infty}\left|\hat{u}_{k}^{n+1}(\omega)\right|^{2} d \omega}
$$

where, $\hat{u}_{k}$ is the final state function in the frequency domain; $\hat{\lambda}$ is the Lagrangian multiplication operator in the frequency domain; $\hat{f}$ is the original signal in the frequency domain.
Step 5. Update $\hat{\lambda}(\omega)$ :

$$
\hat{\lambda}^{n+1}(\omega) \leftarrow \hat{\lambda}^{n}(\omega)+\gamma\left(\hat{f}(\omega)-\sum_{k=1}^{K} \hat{u}_{k}^{n+1}(\omega)\right)
$$

where, $\gamma$ is the noise tolerance coefficient. To ensure the denoising effect, the $\gamma$ value can be set to zero.

Step 6. Repeat Steps 2-4 until the precision $e_{\text {meets the }}$ given convergence constraint $e>0$ :

$$
\sum_{k=1}^{K}\left(\left\|\hat{u}_{k}^{n+1}-\hat{u}_{k}^{n}\right\|_{2}^{2} /\left\|\hat{u}_{k}^{n}\right\|_{2}^{2}\right)<e
$$

Step 7. End the loop and output K components.

\subsection{Autocorrelation function and normalized energy ratio}

The correlations between signals at difference moments can be compared by the autocorrelation function:

$$
R_{x}(\tau)=\lim _{T \rightarrow \infty} \frac{1}{T} \int_{0}^{T} x(t) x(t+\tau) d t
$$

For periodic signals, the autocorrelation function is generally nonzero. For noise signals, the autocorrelation function quickly converges to zero with the growth of time delay. Therefore, the autocorrelation function can be used to evaluate the noise level of signals.

Let $S(t)=\cos (20 \pi t)$ be a signal. After the signal was separately added a noise of $10 \mathrm{~dB}, 5 \mathrm{~dB}, 0 \mathrm{~dB}$, and $-10 \mathrm{~dB}$, the corresponding autocorrelation functions can be calculated as Figure 1.
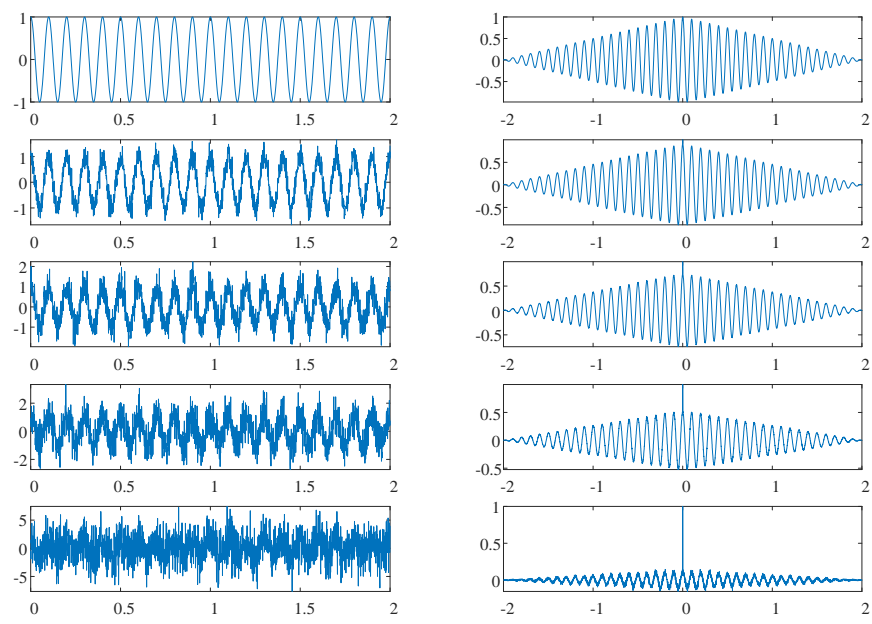

Figure 1. Autocorrelation functions of different noisy signals

As can be seen from Figure 1, the autocorrelation function is an even function of $\tau$, and maximized at 1 when $\tau=0$. The higher the noise level of the signal (except $\tau=0$ ), the closer the function value is to zero.

Then, the normalized energy ratio $\rho_{x}$ can be introduced to better describe the features of the autocorrelation function. Suppose the autocorrelation function of any signal is:

$$
\begin{aligned}
R_{x}(\tau)=\left[R_{x}(-N), R_{x}(-N\right. \\
\left.+1), \cdots, R_{x}(0), \cdots, R_{x}(N)\right]
\end{aligned}
$$


Its energy is:

$$
E_{R x}=\sum_{\tau=-N}^{N} R_{x}^{2}(\tau)
$$

The energy of the autocorrelation function peaks at $E_{R \max }$, when the signal is a constant direct current (DC) signal. Thus, the normalized energy ratio $\rho_{x}$ can be defined as:

$$
\rho_{x}=\frac{E_{R x}}{E_{R \max }}
$$

The $\rho_{x}$ values of the above noisy signals can be calculated as $0.5,0.4149,0.2861,0.1368$, and 0.0076 , respectively.

To sum up, the autocorrelation function and normalized energy ratio help to identify the SNR of each signal. The smaller the SNR, the higher the noise level, and the smaller the $\rho_{x}$. The inverse is also true.

\subsection{Signal reconstruction based on modal energy ratio}

It is assumed that a composite signal contains 4 signals: low-frequency $10 \mathrm{~Hz}$ signal $S_{1}(t), 130 \mathrm{~Hz}$ signal $S_{2}(t), 140 \mathrm{~Hz}$ signal $S_{3}(t)$, and $450 \mathrm{~Hz}$ signal $S_{4}(t)$. The components of the composite signals are either continuous or intermittent in terms of frequency:

$$
\begin{gathered}
S_{1}(t)=0.1 \cos (2 \pi 10 t) \\
S_{2}(t)=0.5 \cos (2 \pi 130 t) \\
S_{3}(t)=0.5 \cos (2 \pi 140 t) \\
S_{4}(t)=\left\{\begin{array}{lr}
0.3 \cos (2 \pi 450 t), & (0 \leq t \leq 0.2 s) \\
0, & (0.2 \leq t \leq 0.4 s) \\
0.3 \cos (2 \pi 450 t), & (0.4 \leq t \leq 0.8 s) \\
0, & (0.8 \leq t \leq 1.1 s) \\
0.3 \cos (2 \pi 450 t), & (1.1 \leq t \leq 1.45 s) \\
0, & (1.45 \leq t \leq 1.7 s) \\
0.3 \cos (2 \pi 450 t), & (1.7 \leq t \leq 2 s)
\end{array}\right) \\
S(t)=S_{1}(t)+S_{2}(t)+S_{3}(t)+S_{4}(t)
\end{gathered}
$$

Figure 2 shows the modals after VMD. Among the six modals, BIMF1, BIMF2, BIMF3, and BIMF6 have basically the same features as the original signal, despite having some noises; BIMF4 and BIMF5 correspond to decomposed noise bands, which are not features of the original signal.

Table 1 lists the $\rho_{x}$ values of the six modal signals obtained by VMD. Figure 3 shows the autocorrelation functions of the modal signals.

As shown in Table 1 and Figure 3, BIMF4 and BIMF5 had far smaller $\rho_{x}$ values (close to zero) than other modals. Their SNRs were very poor. Therefore, the two components must be the decomposed noise bands.

During signal reconstruction, the signals of the two noise modals, namely, BIMFs 4 and 5, were removed to obtain the reconstructed signal and its spectrum (Figure 4).

Table 1. Energy ratio of each modal $(\mathrm{K}=6)$

\begin{tabular}{cccc}
\hline Modal & BIMF1 & BIMF2 & BIMF3 \\
Energy ratio $\rho_{x}$ & 0.3158 & 0.4850 & 0.4928 \\
\hline Modal & BIMF4 & BIMF5 & BIMF6 \\
Energy ratio $\rho_{x}$ & 0.0155 & 0.0135 & 0.1760 \\
\hline
\end{tabular}
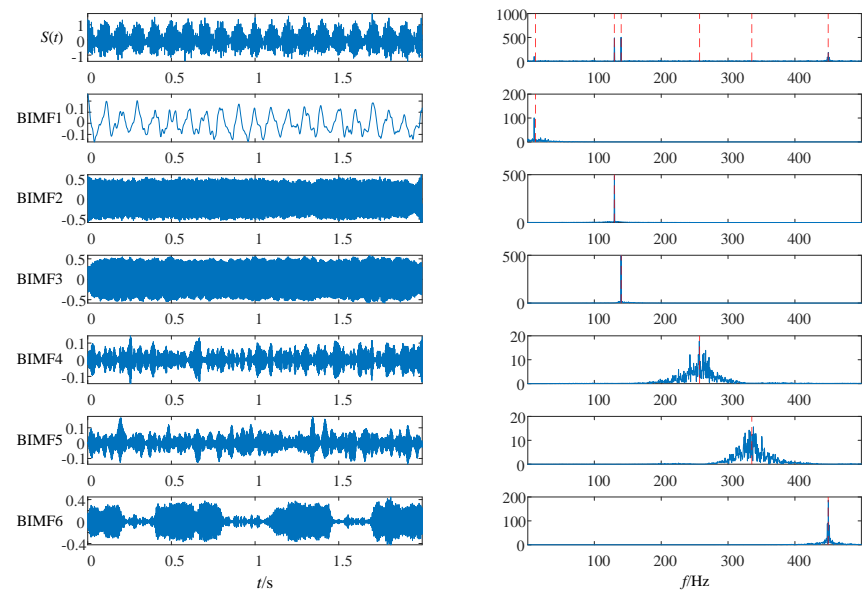

Figure 2. VMD results of noisy signal
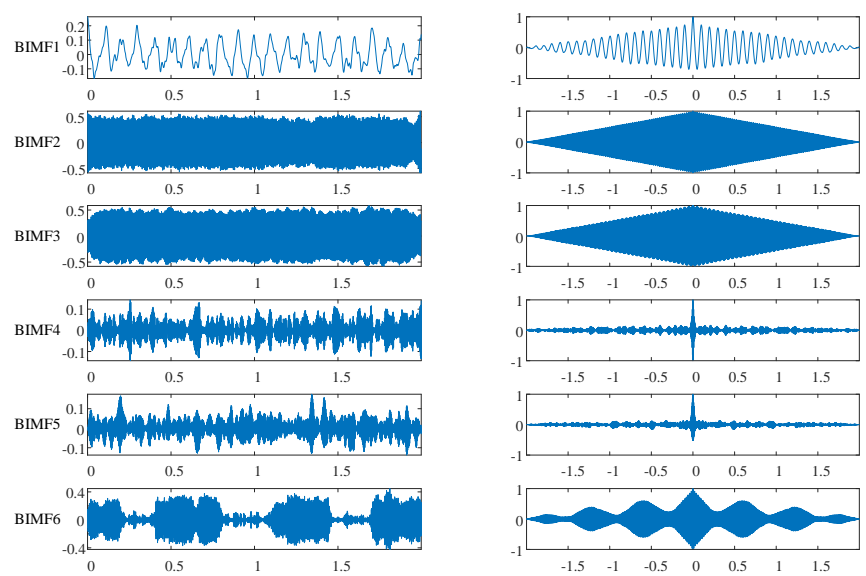

Figure 3. Autocorrelation functions of modal signals

Figure 4 suggests that the reconstructed signal retained the features of the original signal, and contained much fewer noises, due to the removal of the noise bands of BIMF4 and BIMF5. In other words, the reconstructed signal can retain the features of the original signal and eliminates the noises of that signal through the following steps: calculating the normalized energy ratio of the autocorrelation function for each modal acquired by VMD, setting the corresponding threshold, and selecting modals with high SNR for reconstruction.
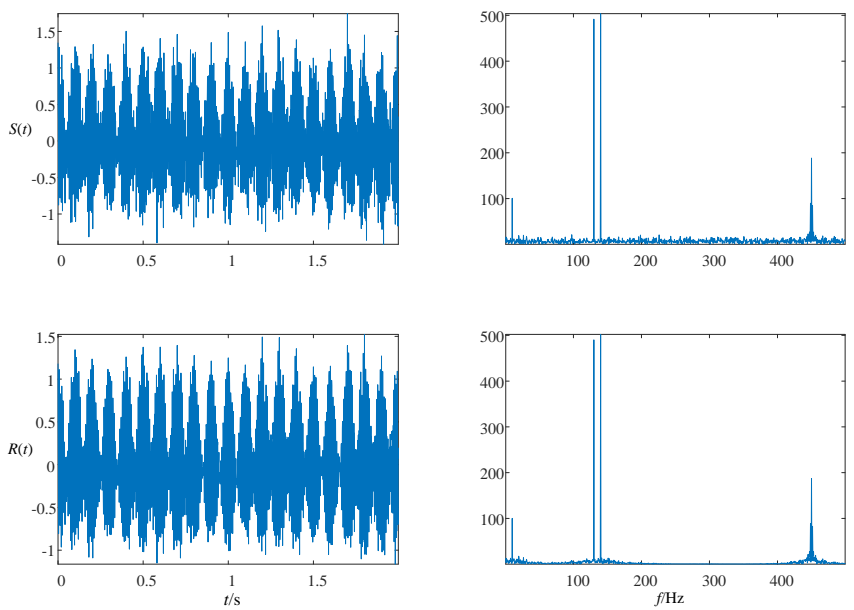

Figure 4. Comparison between reconstructed signal and original signal 
To further verify the denoising effect of the improved VMD in different noise backgrounds, the simulated fault signal (11) was added composite noise signals of $-5 \mathrm{~dB},-10 \mathrm{~dB}$, and $-15 \mathrm{~dB}$, respectively, and then decomposed by the improved VMD, EMD, and LMD in turn. Table 2 presents the SNRs of the signals reconstructed by the three methods.

Table 2. Denoising effects of improved VMD, EMD, and LMD in different noise backgrounds

\begin{tabular}{cccc}
\hline $\begin{array}{c}\text { Decomposition } \\
\text { method }\end{array}$ & $\begin{array}{c}\text { SNproved } \\
\text { VMD }\end{array}$ & LMD & EMD \\
Noise level & 3.3 & -4.24 & -3 \\
$-5 \mathrm{~dB}$ & 1.81 & -9.47 & -7.52 \\
$-10 \mathrm{~dB}$ & -1.08 & -14.38 & -12.05 \\
$-15 \mathrm{~dB}$ & &
\end{tabular}

As shown in Table 2, after the simulated fault signal was added composite white noises with different SNRs, the reconstructed signal saw a decline in its SNR, with the enhancement of noise. Under the three different noise backgrounds, the signal reconstructed by the improved VMD had a far higher SNR than EMD and LMD. Under the additive noise of $-5 \mathrm{~dB}$, the improved VMD led the LMD and EMD by 7.54 and 6.3 in SNR, respectively; under the noise of $-10 \mathrm{~dB}$, the leads increased to 11.28 and 9.33 , respectively; under the noise of $-15 \mathrm{~dB}$, the leads further grew to 13.3 and 10.97 , respectively. In general, the stronger the additive noise, the greater the leads. This means the advantage of the improved VMD in denoising over LMD and EMD increases with the noise intensity.

\section{FCM CLUSTERING}

Taking the weighted sum of the Euclidean distance and fuzzy membership between each data point and each cluster head as the objective function, the FCM clustering gathers similar data points into the clusters through iterative correction of the fuzzy classification matrix and cluster heads, until meeting the convergence constraint at the given precision.

Let $X=\left\{x_{1}, x_{2}, \cdots, x_{\mathrm{n}}\right\}$ be the set of data samples, with $n$ being the number of samples; $V=\left[v_{1}, v_{2}, \cdots, v_{c}\right]^{T}$ be the cluster head vector, with c being the number of cluster heads; $U=\left[u_{i j}\right]_{c \times n}$ be the fuzzy classification matrix, with $u_{i j}$ being the membership of the $\mathrm{j}$-th data point $x_{j}$ relative to the $\mathrm{i}$-th cluster head $v_{i}$. Then, the objective function of the FCM clustering can be defined as:

$$
J(U, V)=\sum_{j=1}^{n} \sum_{i=1}^{c} u_{i, j}^{m} d_{i j}
$$

where, $d_{i j}=\left\|x_{j}-v_{i}\right\|$ is the Euclidean distance from the the $\mathrm{j}$-th data point $x_{j}$ to the $\mathrm{i}$-th cluster head $v_{i} ; m=2$ is the fuzzy weight index. Then, the following constraints can be introduced:

$$
\left\{\begin{array}{l}
0 \leq u_{i j} \leq 1 \\
\sum_{i=1}^{c} u_{i j}=1 \\
\sum_{j=1}^{n} u_{i j}>0
\end{array}\right.
$$

Hence, the FCM clustering mainly iteratively computes $U$ and $V$ to find the minimum interval of the objective function under the above constraints. The process of FCM clustering can be detailed as follows:

Step 1. Define the number of cluster heads c and fuzzy weight index m; initialize fuzzy classification matrix; set the number of iterations $1=0$.

Step 2. Update $v_{i}$ :

$$
v_{i}=\sum_{j=1}^{n} u_{i j}^{m} x_{j} / \sum_{j=1}^{n} u_{i j}^{m}
$$

Step 3. Update U:

$$
u_{i j}=1 / \sum_{k=1}^{c}\left(\frac{d_{i j}}{d_{k j}}\right)^{2 /(m-1)}
$$

Step 4. Repeat Steps 2-4 until the precision $e$ meets the given convergence constraint $e>0$ :

$$
\left\|U^{l+1}-U^{l}\right\|<e
$$

The clustering effect can be evaluated by the classification coefficient $F$ and mean fuzzy entropy $H$ :

$$
F=\frac{1}{n} \sum_{j=1}^{n} \sum_{i=1}^{c} u_{i j}^{2}
$$

$$
H=-\frac{1}{n} \sum_{j=1}^{n} \sum_{i=1}^{c} u_{i j} \ln u_{i j}
$$

The clustering effect improves as the $F$ value approaches 1 and the $H$ value approximates 0 .

\section{EXPERIMENTAL ANALYSIS}

6205-2RS SKF bearings were selected for fault diagnosis experiments. Local damages were made on the bearings manually with an electric discharge machine. Then, vibration acceleration sensors were installed on the casing at the upper end of the motor output support bearing. After that, the vibration signals were measured at different faults, rotation speeds, and load conditions. The vibration signals were analyzed to identify different fault states.

Improved VMD, EMD, and LMD were separately applied to decompose the data on four different states: normal state, inner ring fault, outer ring fault, and ball fault. A total of 100 data samples were collected for each state. The feature parameters of each dataset were calculated, including variance $v$, kurtosis $\beta$, and waveform factor $S_{f}$. The three characteristic quantities of each dataset were compiled into a $400 \times 3$ data matrix, serving as the eigenvector of FCM clustering. The clustering parameters were configured as: the number of cluster heads $c=4$; fuzzy weight index $m=2$; convergence precision $e=0.001$. The clustering results are shown in Figure 5 , where each "." represents a cluster head. 


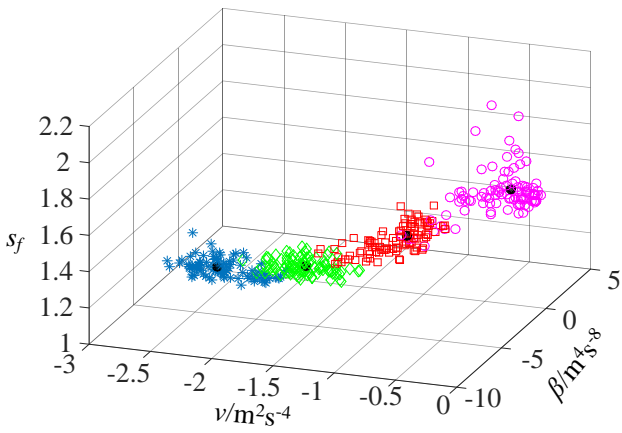

(a) No decomposition

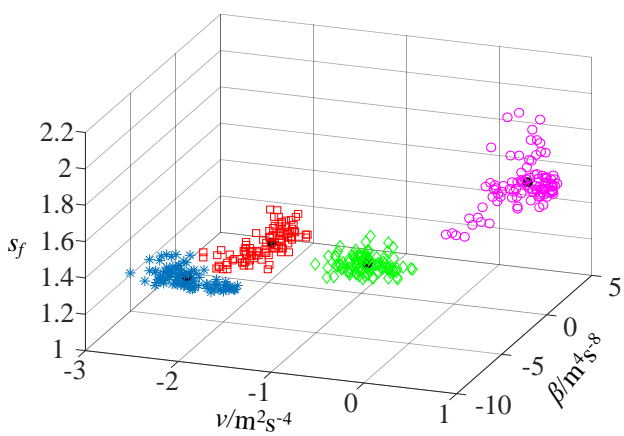

(b) EMD

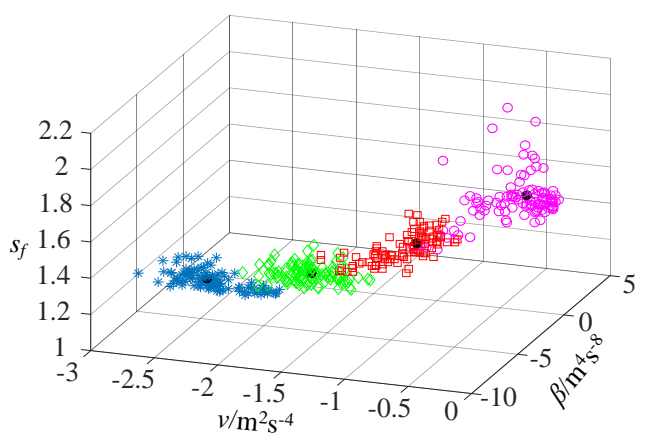

(c) LMD

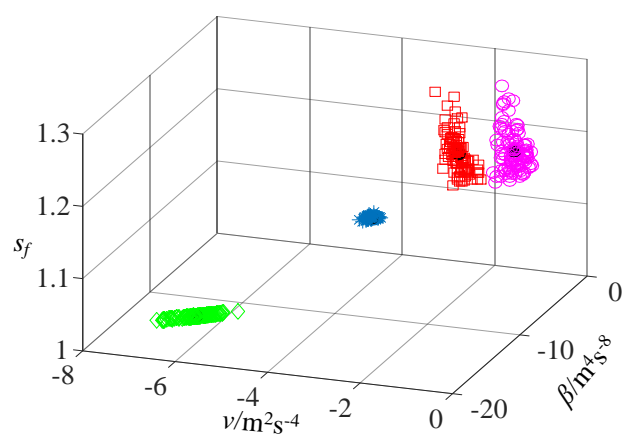

(d) Improved VMD

* Normal state $\square$ Inner ring fault $\diamond$ Ball fault O Outer ring fault

Figure 5. Clustering effects of different decomposition methods

Figure 5 shows that, after being processed by the FCM algorithm, the 100 datasets were not decomposed, or decomposed by EMD, LMD, and improved VMD, respectively. The clustering results of no decomposition, EMD, and LMD cases had two problems: some samples were far from cluster heads; some fault samples of different classes were mixed together. These problems were the most severe in the no decomposition case, where the clustering effect was the worst, adding to the difficulty of fault sample classification. Meanwhile, the fault samples obtained by improved VMD all gathered around the four cluster heads, with clear boundaries between fault classes. Thus, the improved VMD had much better clustering effect than the other three processing approaches.

To further compare the effects of different decomposition methods, the classification coefficient $\mathrm{F}$, and mean fuzzy entropy $\mathrm{H}$ of each method are obtained as shown in Table 3.

Table 3. Fault clustering effects of different decomposition methods

\begin{tabular}{ccccc}
\hline $\begin{array}{c}\text { Clustering } \\
\text { effect } \\
\text { parameters }\end{array}$ & $\begin{array}{c}\text { No } \\
\text { decomposition }\end{array}$ & EMD & LMD & $\begin{array}{c}\text { Improved } \\
\text { VMD }\end{array}$ \\
\hline $\begin{array}{c}\text { Classification } \\
\text { coefficient } F\end{array}$ & 0.8234 & 0.8620 & 0.8376 & 0.9599 \\
$\begin{array}{c}\text { Mean fuzzy } \\
\text { entropy } H\end{array}$ & 0.3483 & 0.2802 & 0.3310 & 0.0955 \\
\hline
\end{tabular}

As shown in Table 3, the improved VMD had the largest F value, which was the closest to 1 . It was higher than the $F$ values of EMD, LMD, and no decomposition by 0.0879 , 0.1223 , 0.1365, respectively. The improved VMD also achieved the minimal $\mathrm{H}$ value, which was the closest to 0 . It was lower than the $\mathrm{H}$ values of EMD, LMD, and no decomposition by $0.1847,0.2455$, and 0.2528 , respectively. These results demonstrate that the improved VMD has a strong denoising effect, i.e., it can effectively remove the noises from the fault signals of rolling bearings. This effect greatly improves the fault detection ability.

\section{CONCLUSIONS}

This paper suggests optimizing and reconstructing the signals decomposed by VMD by modal energy ratio. This strategy can effectively remove noises from noisy signals, while retaining the fault features. The stronger the noise, the more superior the improved VMD is to LMD and EMD. Next, the eigenvectors of sample signals were classified and recognized through FCM clustering. Then, the clustering effect of our method was compared with that of no decomposition, EMD, and LMD. The comparison shows that the fault samples obtained by the improved VMD all gathered around the four cluster heads, with clear boundaries between fault classes. Thus, the improved VMD had much better clustering effect than the other three processing approaches. Based on the improved VMD and FCM clustering, the proposed rolling bearing fault recognition method overcomes the difficulty in extracting the fault features of rolling bearings in the background of composite noises, and greatly improves the fault detection ability for rolling bearings.

\section{ACKNOWLEDGMENT}

This work was partially supported by the National Natural Science Foundation of China (Grant No.: 61403329, 61503213) and Natural Science Foundation of Zhejiang Province (Grant No.: LGN20C050002). 


\section{REFERENCES}

[1] Jiang, W.L., Wang, H.N., Zhu, Y., Wang, Z.W., Dong, K.Y. (2017). Integrated VMD denoising and KFCM clustering fault identification method of rolling bearings. China Mechanical Engineering, 28(10): 1215-1220, 1226. https://doi.org/10.3969/j.issn.1004132X.2017.10.013

[2] Shi, P.M., Wang, J., Wen, J.T., Tian, G.J. (2016). Study on rotating machinery fault diagnosis method based on envelopes fitting algorithms EMD. Journal of Metrology, (1): 62-66. https://doi.org/10.3969/j.issn.10001158.2016.01.15

[3] Yang, Y., Cheng, J., Zhang, K. (2012). An ensemble local means decomposition method and its application to local rub-impact fault diagnosis of the rotor systems. Measurement, 45(3): $561-570$ https://doi.org/10.1016/j.measurement.2011.10.010

[4] Jiang, H., Chen, J., Dong, G., Liu, T., Chen, G. (2015). Study on Hankel matrix-based SVD and its application in rolling element bearing fault diagnosis. Mechanical Systems and Signal Processing, 52: 338-359. https://doi.org/10.1016/j.ymssp.2014.07.019

[5] Zheng, J., Cheng, J., Yang, Y. (2013). A rolling bearing fault diagnosis approach based on LCD and fuzzy entropy. Mechanism and Machine Theory, 70: 441-453. https://doi.org/10.1016/j.mechmachtheory.2013.08.014

[6] Yang, Q., Chen, G.M., He, Q.F., Liu, Q.J. (2012). Application of local tangent space permutation algorithm in bearing early fault diagnosis. Journal of Vibration Measurement \& Diagnosis, 32(5): 831-835. https://doi.org/10.3969/j.issn.1004-6801.2012.05.025

[7] Zolfaghari, S., Noor, S.B.M., Rezazadeh Mehrjou, M., Marhaban, M.H., Mariun, N. (2018). Broken rotor bar fault detection and classification using wavelet packet signature analysis based on fourier transform and multilayer perceptron neural network. Applied Sciences, 8(1): 25. https://doi.org/10.3390/app8010025

[8] Wang, J.G., Wu, L.F., Qin, X.H. (2014). Rolling bearing vibration signal fault feature extraction based on autocorrelation analysis and LMD. China Mechanical Engineering, 25(2): 186-190. https://doi.org/10.3969/j.issn.1004-132X.2014.02.009

[9] Cheng, J., Yang, Y., Yang, Y. (2012). A rotating machinery fault diagnosis method based on local mean decomposition. Digital Signal Processing, 22(2): 356-
366. https://doi.org/10.1016/j.dsp.2011.09.008

[10] Li, Y., Xu, M., Wang, R., Huang, W. (2016). A fault diagnosis scheme for rolling bearing based on local mean decomposition and improved multiscale fuzzy entropy. Journal of Sound and Vibration, 360: 277-299. https://doi.org/10.1016/j.jsv.2015.09.016

[11] MenG, Z., Gu, W.M., Hu, M., Xiong, J.M. (2016). Fault feature extraction of rolling bearing incipient fault based on improved singular value decomposition and EMD. Acta Metrologica Sinica, 37(4): 406-410. https://doi.org/10.3969/j.issn.1000-1158.2016.04.16

[12] Dong, G., Chen, J. (2012). Noise resistant time frequency analysis and application in fault diagnosis of rolling element bearings. Mechanical Systems and Signal Processing, 33: 212-236. https://doi.org/10.1016/j.ymssp.2012.06.008

[13] Wang, H., Chen, J., Dong, G. (2014). Feature extraction of rolling bearing's early weak fault based on EEMD and tunable Q-factor wavelet transform. Mechanical Systems and Signal Processing, 48(1-2): 103-119. https://doi.org/10.1016/j.ymssp.2014.04.006

[14] Dragomiretskiy, K., Zosso, D. (2013). Variational mode decomposition. IEEE Transactions on Signal Processing, 62(3): 531-544. https://doi.org/10.1109/TSP.2013.2288675

[15] Ma, Z.Q., Li, Y.C., Liu, Z., Gu, C.J. (2016). Rolling bearings' fault feature extraction based on variational mode decomposition and Teager energy operator. Vibration and Shock, 35(13): 134-139. https://doi.org/10.13465/j.cnki.jvs.2016.13.022

[16] Gupta, K.K., Raju, K.S. (2014). Bearing fault analysis using variational mode decomposition. In 2014 9th International Conference on Industrial and Information Systems (ICIIS), Gwalior, India, pp. 1-6. https://doi.org/10.1109/ICIINFS.2014.7036617

[17] Chang, Y., Bao, G.Q., Chen, S.K., Chen, P. (2020). Optimization and research of a bearing fault diagnosis method based on VMD and KFCM. Journal of Southwest University, $\quad 42(10)$ : 146-155. https://doi.org/10.13718/j.cnki.xdzk.2020.10.019

[18] Vishnoi, S., Jain, A.K., Sharma, P.K. (2019). An efficient nuclei segmentation method based on roulette wheel whale optimization and fuzzy clustering. Evolutionary Intelligence, 1-12. https://doi.org/10.1007/s12065-01900288-5 